\title{
INNOVACIÓN ABIERTA EN LA FACULTAD DE INGENIERÍA
}

Marcos Lavandera ${ }^{1}$, Joaquín Domato ${ }^{2}$, Esteban Seibel $^{3}$

\section{RESUMEN}

En el año 2012, estudiantes de la facultad de ingeniería de Olavarría participaron por iniciativa propia del evento/ competencia internacional " $24 \mathrm{hs}$ de Innovación", organizado en conjunto por universidades de Francia y Canadá.

Este evento se basa en el formato Charrette de trabajo (Proyecto rápido, repentino o rally de Innovación) donde los participantes organizados en equipos, tienen 24 hs reloj para proponer una solución creativa e innovadora a los desafíos propuestos por instituciones privadas y públicas de los diferentes países participantes.

En los años 2013, 2014 y2015, la Facultad de Ingeniería fue una de las sedes de este evento. Esto significó para la facultad, la posibilidad de ser el lugar de encuentro en donde los estudiantes pueden desafiarse a encontrar soluciones a problemas reales de las empresas y organismos públicos que participan. En el 2014 se suman estudiantes de los últimos años de las escuelas secundarias.
Con estos antecedentes y los buenos resultados obtenidos en términos de participación y aprendizaje vivencial e innovador, la Facultad de Ingeniería ha decidido replicar estas competiciones para estudiantes del último año de escuelas secundarias, en el marco del convenio con la Fundación Loma Negra y a través del Proyecto de Extensión "Para Emprendedores". Creando así el evento "24 hs de Innovación FIO".

Las "24 horas de Innovación FIO" es una competencia, basada en la metodología de Innovación Abierta, que se desarrolla en 24 horas consecutivas durante las cuales se deben generar proyectos rápidos, repentinos e innovadores que permitan resolver problemáticas de índole público o privado.

Se logra así, motivar a los estudiantes para que dentro de sus opciones a futuro se encuentren los estudios superiores, entren en contacto con la Facultad y adquieran competencias mediante aprendizaje vivencial para su desarrollo académico y/o profesional.

A partir de los resultados obtenidos,

\footnotetext{
${ }^{1}$ Ingeniero Químico y Magister en Administración de Negocios. Jefe de Trabajos Prácticos Ordinario, Dpto. de Ing. Industrial. Director del Proyecto de Extensión "Para Emprendedores” y Director del Centro de Desarrollo Emprendedor e Innovación (CDEI) de la Facultad de Ingeniería de Olavarría. Universidad Nacional del Centro de la Provincia de Buenos Aires (UNICEN). Argentina. E-mail: marcos@fio.unicen.edu.ar.

${ }^{2}$ Estudiante de Ingeniería Industrial en la Facultad de Ingeniería de Olavarría. Universidad Nacional del Centro de la Provincia de Buenos Aires (UNICEN). Argentina. Miembro del Proyecto de Extensión "Para Emprendedores". Email: domatomjoaquin@gmail.com

3 Estudiante de Ingeniería Industrial en la Facultad de Ingeniería de Olavarría. Universidad Nacional del Centro de la Provincia de Buenos Aires (UNICEN). Argentina. Miembro del Proyecto de Extensión "Para Emprendedores". Email: este.seibel@gamail.com
} 
debieran iniciarse instancias de protoinvestigación que conduzcan a nuevas acciones en clave de integralidad (docencia - investigación - extensión). El impacto de estos procesos es inmediato si existe la decisión política y los apoyos institucionales que promuevan distintas formas de incorporar conocimientos y adquirir competencias, transgrediendo lo instituido.

\section{INTRODUCCIÓN}

A lo largo de muchos años las grandes organizaciones han desarrollado modelos de innovación de una manera cerrada e integrada por agentes internos, dejando a un lado la colaboración de agentes externos y fomentando así el modelo tradicional de innovación. Este modelo se caracteriza por una lógica de profundo desarrollo interno, donde las labores de investigación, desarrollo y comercialización de las innovaciones se realizan desde dentro de la empresa, creando y transfiriendo conocimiento internamente, evitando dicha transferencia hacia los competidores (Chesbrough, 2003). A las empresas tradicionales les ha resultado ventajoso usar este modelo amparadas en la ventajas competitivas adquiridas en relación a otras que venían relegadas en estos aspectos, éstas últimas y el avance de las tecnologías de la información y la comunicación (TIC's), obligan a nuevas procesos de innovación y desarrollo, incorporando la idea de la "empresa extendida", vinculando al entorno con las problemáticas internas.

Por otro lado, en los últimos años se vienen dando cambios sociopolíticos que han debilitado de cierta manera la efectividad del sistema de innovación tradicional. El auge de la sociedad del conocimiento junto con la evolución protagónica de los medios masivos de comunicación, el aumento del número de trabajadores altamente calificados y su mayor movilidad, la mayor tasa de obsolescencia y nivel de complejidad de las tecnologías junto a la existencia de un stock de conocimiento básico en el interior de las empresas con múltiples usos, han propiciado la necesidad de detectar y explotar las ideas valiosas, independientemente de dónde surjan (De Jong, 2008). Chesbrough señala que las tecnologías actuales pueden ser demasiado complejas para ser desarrolladas por un mismo equipo de investigación, por lo que las empresas deben aprender a cooperar entre sí y fomentar la participación de áreas ajenas a estas para aprovechar la experiencia que cada uno tiene. De esta manera se busca una nueva estrategia de innovación bajo la cual las empresas van más allá de los límites internos de su organización y donde la cooperación con profesionales externos pasa a tener un papel fundamental.

Michael Porter afirma que la competitividad de una nación, en la cual se sustentan su desarrollo industrial y su soberanía económica, depende de la capacidad para innovar e implementar nuevas y mejores técnicas. Las actividades de innovación constituyen junto con el capital humano uno de los principales factores que determinan las ventajas competitivas de las economías industriales avanzadas.

Se observa así, que la clave para el desarrollo de las empresas del futuro está en la innovación abierta. Estas deberán aprender a mejorar o incluso cambiar su modelo o sistema organizacional, considerando que no todas las buenas ideas están dentro de una única empresa, sino que por el contrario se pueden obtener o combinar con ideas de distintas partes del mundo, y solo de esta manera las empresas podrán innovar con éxito, sustentándose en las claves para las empresas del futuro mencionadas por Chesbrough: ser abiertas, aceptar el fracaso e innovar tanto en el modelo de negocio así como también en la tecnología, sin 
dejar a un lado los pilares fundamentales de la innovación como la creatividad (un trabajo multidisciplinar por naturaleza), el pensamiento disruptivo o divergente y una perspectiva sistémica surgida del trabajo en equipo. La innovación abierta requiere colaboración e implica articular esfuerzos entre organizaciones, fomentando la creación de un ecosistema de innovación con compromiso territorial, interpelado por la responsabilidad social empresarial, en el proceso innovador que permita integrar el conocimiento interno y externo.

Cabe destacar que estos conceptos mencionados no solo son utilizados por el sector privado, sino que por su efectividad y dinamismo han sido trasladados al sector público. En los últimos años, debido al incremento de políticas destinadas a fomentar la industria nacional, han proliferado la creación de emprendimientos y pequeñas empresas. En este marco, muchos de estos emprendimientos carecen de la estructura necesaria para ser competitivos en los contextos continuamente variables de la actualidad, derivando así en una alta tasa de mortalidad. Por esta razón, se crean programas para el desarrollo de emprendimientos de calidad en la región motivando y concientizando así a los emprendedores que deben innovar en sus productos o servicios, para lograr adaptarse al mercado.

Bajo este paradigma, la Facultad de Ingeniería de Olavarría (FIO) de la Universidad Nacional del Centro de la Provincia de Buenos Aires (UNCPBA) ha adoptado en los últimos años una política que contribuye con la cultura emprendedora en la formación profesional de los futuros ingenieros a través de capacitaciones y asistencia técnica. A partir de esta política dentro de la Facultad se desarrolla el Proyecto de Extensión "Para Emprendedores" (PEPE) cuyo objetivo general es: Fomentar la cultura emprendedora facilitando la generación de nuevos emprendimientos y fortaleciendo los ya existentes, a través de un espacio inclusivo, colaborativo, interdisciplinario e interinstitucional, dentro del ámbito universitario, para el apoyo a emprendedores que contribuyan al desarrollo económico regional con igualdad de oportunidades y equidad social.

En el marco de este proyecto se han realizado actividades de tutoría a emprendedores de la región, reuniones y capacitaciones en consultoría para los estudiantes y se participa y organizan actividades de innovación abierta, teniendo en cuenta la importancia de la temática a nivel mundial y regional para la formación y capacitación de emprendedores que logren competir en los contextos actuales.

Por iniciativa propia de estudiantes de la FIO en el año 2013 se participó de forma espontánea en una competencia internacional llamada "Les 24h de l'innovation" (Las 24 hs de Innovación). La misma está basada en el formato denominado Charrete de trabajo (proyecto rápido, repentina o rally de innovación), donde por equipos, los participantes deben emplear todo su ingenio y creatividad para en tan solo 24 hs brindar ideas para soluciones originales a los desafíos propuestos por empresas privadas, instituciones públicas y ciudadanos, entre otros.

A partir de esta primera experiencia, se realizaron las gestiones correspondientes para que en el mes de mayo del año 2014, la FIO se convirtiese en una de las sedes oficiales del evento en Argentina. Luego en el mes de octubre del mismo año, se participó de otra competencia con la misma metodología denominada "Rally latinoamericano de Innovación", promovida por el Consejo Federal de Decanos de Ingeniería (CONFEDI) donde los desafíos son propuestos tanto por empresas privadas como instituciones públicas de Latinoamérica. 
Luego, se decidió trasladar esta metodología de aprendizaje y competencia al ámbito preuniversitario, principalmente a los estudiantes del último año de escuelas secundarias, tanto de técnicas como no técnicas y de gestión estatal como gestión privada. Se propuso un evento organizado por el PEPE y la Facultad de Ingeniería en colaboración con Fundación Loma Negra, la Agencia de Desarrollo Económico Local de Olavarría y Centro de Innovación y Creación de Empresas de la Universidad, donde a los estudiantes secundarios se los invita a participar en una competencia basada en la metodología mencionada anteriormente, donde los desafíos son problemáticas reales de empresas privadas e institucionespúblicasdeOlavarríaylaregión.

Nacen así las "24 Hs de Innovación FIO" cuyo objetivo general es: Fomentar la interrelación de los estudiantes de escuelas secundarias con la Facultad de Ingeniería y los distintos agentes locales, resolviendo problemáticas de interés Público y Privado, generando espacios interdisciplinarios que permitan desarrollar la creatividad y la formulación de ideas, a través del concepto de innovación abierta

De esta manera, este trabajo describe cómo el aprendizaje por experiencia, a través de la metodología de innovación abierta, permite desarrollar competencias y aptitudes emprendedoras en estudiantes de escuelas secundarias, las cuales están vinculadas a la política adoptada por la FIO.

\section{ASPECTOSTEÓRICOS (FUN- DAMENTACIÓN)}

El profesor Henry Chesbrough, define la innovación abierta como el uso de los flujos internos y externos de conocimiento para acelerar la innovación inter- na. Este tipo de innovación permite a las organizaciones un constante aprendizaje, brindándoles adaptabilidad a un entorno cambiante. En palabras del profesor: " las organizaciones que tienen éxito a menudo terminan atrapadas en su modelo. Es necesario aprender cómo mejorar o incluso cambiar este modelo para seguir prosperando una vez que se vuelva obsoleto."

Los procesos de innovación abierta dentro de las organizaciones tienen como objetivo el acceso a ideas internas y externas para crear valor y lograr mantenerlo mediante mecanismos internos. Henry Chesbrough, define además, dos modelos principales por el que fluyen las ideas, "Fuera - Dentro" y "Dentro - Fuera". En este sentido, la idea de "fuera - dentro" implica abrir la organización a muchos tipos de información y aportaciones externas, mientras que "dentro - fuera" requiere que las instituciones permiten que las ideas propias no utilizadas salgan al exterior para que otros puedan utilizarlas.

Se define además la innovación abierta como la metodología que aplican las organizaciones, de todos los ámbitos, para poder mejorar, crecer y encontrar soluciones innovadoras a los problemas que se presentan diariamente, a partir de información externa. Este modelo, implica una relación de vínculo entre las organizaciones y los agentes externos a estas, ya sean profesionales o no, en búsqueda de inteligencia colectiva.

La innovación abierta se transforma así en un proceso de aprendizaje organizacional. Esta metodología es extrapolable a todos los sistemas de enseñanza - aprendizaje. E. Litwin (1997) critica el sistema tradicional de la enseñanza cuando dice: "Al analizar las prácticas de la enseñanza, otro de los problemas que distinguimos con frecuencia es el carácter no auténtico del 
discurso pedagógico. Esto último se refiere al perfil de los problemas, que si bien en apariencia guardan relación de semejanza con problemas reales, no presentan ni la complejidad ni el juego de variables o determinaciones que pertenecen al mundo de la vida real". El desarrollo de la capacidad de análisis crítico y de aplicación del conocimiento es de fundamental importancia en la formación del estudiante de cualquier nivel académico por lo que es necesario incorporar nuevas metodologías que ayuden a una formación en competencias y habilidades adaptadas a los nuevos entornos.

La utilización del modelo de innovación abierta en el ámbito de la educación permite generar espacios de aprendizaje dentro de escenarios basados en la realidad. Esta metodología logra vincular a los estudiantes con problemas concretos, buscando soluciones factibles surgidas de la reflexión y comprensión de su contexto.

A partir de este concepto, aplicado en los distintos niveles educativos se presenta una manera alternativa de aprendizaje, tanto para los alumnos como para los docentes, en donde se desarrollan competencias propia de una metodología activa de aprendizaje. De esta manera se encuentra una forma de enfocar el aprendizaje a todos los actores participantes de la educación (Estudiantes y Docentes). Con el desarrollo de esta metodología dentro o fuera de las instituciones educativas, enfocándose en el aprendizaje por experiencia, descripto por Kolb (1984, citado en Alonso, et al., 1997): "algunas capacidades de aprender que se destacan por encima de otras como resultado del aparato hereditario de las experiencias vitales propias y de las exigencias del medio ambiente actual..." riencial se adapta a las nuevas demandas profesionales vinculadas con la formación y desarrollo de competencias y habilidades, generando además un proceso reflexivo para la construcción del conocimiento a partir de la experiencia realizada.

Dentro de estas estrategias de aprendizaje significativo se encuentran (Itin 1999):

- Solución a problemas reales

- Análisis de casos (Análisis y aplicación de conocimientos,tomas de decisiones)

- Aprendizaje basado en proyectos

- Trabajo en grupo

- Simulaciones de contexto reales

- Aprendizajes mediados por TICs

Para Kolb (1981) el aprendizaje es "el proceso mediante el cual se crea conocimiento a través de la transformación de la experiencia". Propone un modelo de aprendizaje formado por cuatro etapas que se relacionan:

- Experiencia concreta

- Observación reflexiva

- Conceptualización abstracta

- Experimentación activa

Estas etapas describen un ciclo donde se relaciona la experiencia con la reflexión para la formación de conceptos abstractos. En las cuatro fases del aprendizaje la "experiencia" es la base para la "observación y reflexión”. Luego las observaciones son asimiladas formando un nuevo grupo de "conceptos abstractos" y "generalizaciones" de la que se deducen nuevas implicaciones para la acción.

Esta propuesta de aprendizaje expe- 


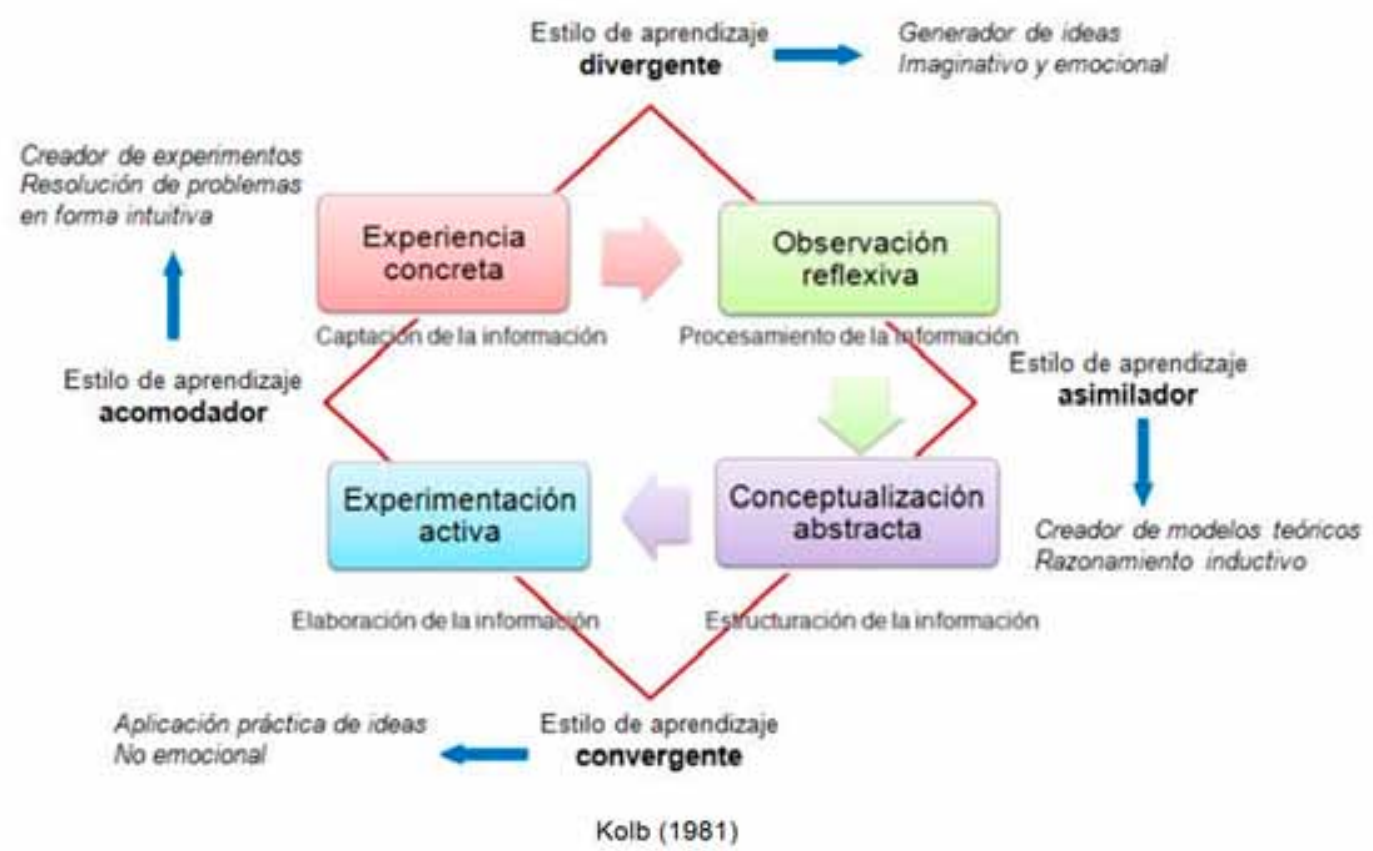

Como se puede ver a partir del ciclo de aprendizaje de Kolb, este comienza a partir de una experiencia concreta, una experiencia vivida por el individuo (estudiante para el objeto de este trabajo) por lo que habría que analizar, si todas las experiencias vividas generan aprendizaje.

Para lograr esto es necesario articular los conocimientos previamente adquiridos con la experimentación activa, formando una estructura conceptual que permita aplicar el conocimiento generado desde una experiencia concreta a nuevas situaciones.

Itin (1999) propone un modelo que integra las fases de Kolb y en el cual el aprendizaje experiencial es un proceso formativo que implica al individuo físicamente, socialmente, intelectualmente, cognitiva $y$ emocionalmente a través de una experiencia concreta que le ofrece un reto con un nivel de riesgo y fracaso. En este proceso los estudiantes formulan hipótesis, experimentan y utilizan la creatividad desarrollando conocimientos.

Este proceso se sustenta en lo que se conoce como "estado de flujo" (Mihály Csíkszentmihályi, 1975), que se puede definir como el estado en el que se integran el disfrute y el esfuerzo en un equilibrio dinámico, un estado en el que las personas involucradas se encuentran tan comprometidas con las tareas que nada les parece más importante.

En este Modelo del Reto Óptimo convergen el nivel de dificultad de la actividad o tarea, y las habilidades de las que dispone la persona para resolver la situación problemática. La activación motivacional no depende sólo de la novedad o interés intrínseco del trabajo en cuestión, sino de la correspondencia entre ésta y los recursos personales de los que se dispone para afrontar la situación en un equilibrio entre la competencia del individuo y el desafío implicado en la tarea. De forma resumida, diremos que según Csikszentmihalyi, la 
experiencia de flujo requiere un equilibrio óptimo entre los desafíos percibidos y las habilidades del sujeto. Cuando no existe ese equilibrio, podemos tener dos situaciones diferentes: si los desafíos superan las competencias individuales se genera un estado de ansiedad por exceso de dificultad, mientras que si, por el contrario, las habilidades superan demasiado los desafíos colocados, el individuo estará aburrido y, por ende, poco motivado. Este equilibrio lo podemos ver representado en la siguiente figura, propuesta por Whalen (1997), donde existirá un "canal de flujo" en el área de equilibrio óptimo entre los factores de las habilidades del sujeto y el desafío percibido para la realización de la tarea.

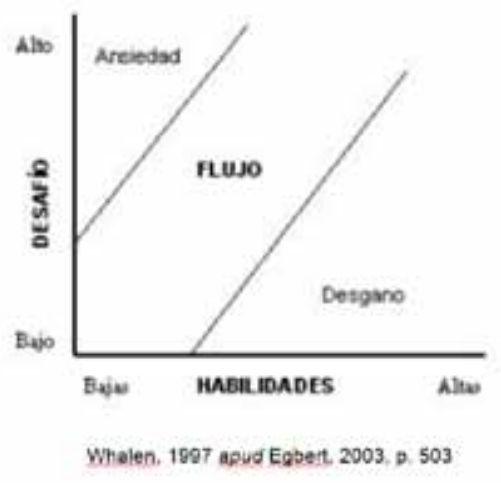

Csikszentmihalyi (1992), alerta sobre el hecho que no se debe describir el flujo unidimensionalmente a través del equilibrio percibido entre desafíos y habilidades, ya que al trabajar con la motivación de los participantes, el flujo "es un fenómeno complicado basado en la interacción entre los aprendices, las tareas y las variables contextuales"

De esta manera, el éxito de esta forma de aprendizaje radica en que cada participante construye conocimientos y competencias a partir de sus fortalezas y debilidades, logrando un sentido de titularidad respecto de lo aprendido. Esto se sustenta en que los problemas y los escenarios que se presentan tienden a ser poco convencionales, generando condiciones de integración grupal e interdependencia de los participantes que no se encuentran en otros ámbitos educativos, lo que, sumado a la percepción de riesgo, predispone al grupo al aprendizaje. Como corolario de este proceso encontramos que las acciones individuales y grupales fomentan la búsqueda de nuevos paradigmas de pensamiento y conducta, dando como resultado un aprendizaje generalizable, ya que los comportamientos manifestados durante las actividades son los mismos que se exteriorizan en otros escenarios personales y laborales.

\section{DESARROLLO}

\section{Hs de Innovación:}

Las "24 horas de Innovación" es un evento que se lleva a cabo a nivel internacional desde hace ya 5 años, en el formato de Charrete de trabajo (proyecto rápido, repentino o rally de innovación). Durante el mismo, los participantes deben emplear todo su ingenio y creatividad para en tan solo 24 hs brindar soluciones originales a los desafíos propuestos por empresas privadas, instituciones públicas y ciudadanos, entre otros. Para luego presentarlas a través de un video de 2 minutos. 
Este tipo de competencia está orientada a estudiantes universitarios, preuniversitarios y profesionales de cualquier disciplina.

Las "24 horas de Innovación” se realizan anualmente en Francia (octubre) y en $\mathrm{Ca}$ nadá (mayo). Fueron fundadas por Jéremy Legardeur y su equipo en la ESTIA Ecole Superieuredes Technologies Industrielles Avancées en Francia y la versión que se realiza en Québec por Mickael Gardoni de la Ècole de tecnologie supérieure ETSMontreal.

Durante 2013 y 2014 la Facultad de Ingeniería de la Universidad Nacional del Centro de la Provincia de Buenos Aires ha organizado la participación de equipos de trabajo interdisciplinarios compuestos por docentes, graduados y estudiantes.

La primera participación en el 2013 fue de forma espontánea, un grupo de estudiantes, por iniciativa propia, dieron a conocer el evento en la facultad y formaron un equipo de trabajo para participar. A partir de esta primera experiencia, se realizaron las gestiones correspondientes a través de la Fundación Redes de Conocimiento (Coordinadora del evento en la Argentina) para que en el mes de mayo 2014, la FIO se convirtiese en una de las sedes oficiales del evento en Argentina. En esta ocasión concurrieron al evento aproximadamente 180 personas, de las cuales 80 participaron de la competencia, formando 9 equipos de trabajo. Además fue la única sede que contó con la participación de estudiantes de nivel secundario, con 30 jóvenes de distintas escuelas. De esta primera experiencia como organizadores se puede destacar que la investigación, innovación abierta, trabajo en equipo y la comunicación se convirtieron en piezas claves para el desarrollo del evento.
A su vez además de los participantes, concurrieron 15 profesores y profesionales, personal de apoyo y 10 estudiantes universitarios que forman parte del Proyecto de Extensión "Para Emprendedores", que fueron parte de la organización.

De manera simultánea con la competencia se brindaron talleres culturales y de capacitación para dar a conocer herramientas útiles en este tipo de eventos, y actividades disruptivas como partidos de ping pong, cena informal, encuentro de bandas y otros espectáculos.

Al año siguiente, en el mes de mayo de 2015, la FIO volvió a ser sede oficial del evento y esta vez participaron 70 personas, entre concursantes y organizadores, de distintas carreras universitarias y terciarias, además de alrededor de 20 estudiantes del nivel secundario.

\section{RALLY LATINOAMERICANO DE INNOVACIÓN 2014:}

\section{El "RALLY LATINOAMERICANO} DE INNOVACIÓN" es una competencia internacional que tiene como propósito fomentar la innovación abierta en estudiantes de carreras de ingeniería de Latinoamérica y que se desarrolla por equipos durante 28 horas consecutivas. La primera edición se llevó a cabo los días 17 y 18 de octubre de 2014.

El Rally nace en el marco del Programa Regional de Emprendedorismo e Innovación en Ingeniería (PRECITYE) y toma como referencia y está inspirado en la competencia denominada "The 24 bs of innovation" creada en el año 2007 por el Instituto de Ingeniería ESTIA (Ecole Superieure des Technologies Industrielles Avancées) de Francia. Partiendo del formato original, desde el 
PRECITYE se diseñó este nuevo formato que convoca a estudiantes de las Facultades de Ingeniería de Latinoamérica, en forma conjunta con estudiantes de otras facultades con el propósito de promover una nueva cultura de innovación abierta y contribuir con nuevas propuestas de solución a problemas reales de la región.

La competencia propone resolver desafíos que consistirán en problemas reales que requieran de una solución creativa, no estando limitados únicamente al ámbito tecnológico, pudiendo ser de varios sectores de actividades o temas sociales, ambientales, organizacionales, artísticos, logísticos o de otro tipo.

Las facultades de ingeniería convocarán a equipos de estudiantes de ingeniería a participar del Rally de Innovación, donde se les presentarán los desafíos enviados por las instituciones y empresas. Está previsto y se promueve que además de los estudiantes de ingeniería sean convocados estudiantes de otras disciplinas, conformando equipos multidisciplinarios para el abordaje de los desafíos planteados.

Los equipos trabajarán físicamente en las sedes localizadas en las universidades anfitrionas o en los lugares que éstas, en potencial asociación con otras instituciones, determinen como sedes físicas.

Los equipos tienen 28 horas para seleccionar un desafío, entender el problema, conformar los equipos, plantear una solución, identificar los beneficiarios, validarla, ajustar lo que consideren necesario hasta lograr una propuesta que será presentada mediante un video con una duración de hasta 3 minutos y un reporte pautado (que incluye un análisis vía CANVAS) que da cuenta de la potencial sustentabilidad e impacto social de la propuesta. Todo el material entregado por los equipos pasará a formar parte del dominio público.

Los jurados de cada sede analizan las propuestas recibidas y proclaman ganadores en dos categorías:

- Innovación: propuestas que se destaquen en aspectos de innovación.

- Impacto social: propuestas que se destaquen por su impacto social.

En su primera edición en el año 2014, la FIO fue sede oficial de la competencia albergando a estudiantes de la FIO, de la Facultad de Ingeniería de la Universidad FASTA, estudiantes de Ciencias Económicas y de la carrera de Licenciatura de Comunicacion Social de la Facultad de Ciencias Sociales de Olavarría. (58 participantes de Mar del Plata, Olavarría, Tandil y Bahía Blanca).

En esta edición se formaron 6 equipos, constituidos por estudiantes de Ingeniería Industrial, Ingeniería Civil, Ingeniería Química, Ingeniería Informática, Ingeniería Ambiental, Ingeniería Electromecánica, Licenciatura en comunicación social y Contaduría Pública. Siendo ganadores en Innovación la propuesta de un equipo constituido por Estudiantes de Ingeniería Informática y Licenciatura en Comunicación Social, y en Impacto Social el equipo de Ingeniería Industrial e Ingeniería Ambiental.

\section{HS DE INNOVACIÓN FIO}

En este apartado se describe la propuesta de la FIO donde se aplicó la metodología de innovación abierta con estudiantes de escuelas secundarias. 
Con estos antecedentes y los buenos resultados obtenidos en términos de participación y aprendizaje vivencial e innovador, la Facultad de Ingeniería ha decidido replicar estas competiciones pero con desafíos locales para estudiantes del último año de escuelas secundarias, en el marco del convenio con la Fundación Loma Negra y a través del Proyecto de Extensión "Para Emprendedores". Entendemos esta actividad como una forma inédita de vinculación entre las empresas, la universidad, el sector educativo secundario y el estado, donde los estudiantes podrán tomar contacto con los distintos sectores productivos de la sociedad y creativamente contribuir a la solución de temáticas tanto públicas como privadas. Para ello desde la facultad se decide desarrollar el evento denominado " 24 hs de innovación FIO”.

Las 24 horas de Innovación FIO es una competencia, basada en la metodología de Innovación Abierta, que se desarrolla en 24 horas consecutivas durante las cuales se deben generar proyectos rápidos, repentinos e innovadores que permitan resolver problemáticas de índole público o privado. Los destinatarios de este evento son los estudiantes del último año de las escuelas de educación secundaria de Olavarría y la zona.

\section{Objetivo General:}

Fomentar la interrelación de los estudiantes de escuelas secundarias con $\mathrm{la} \mathrm{Fa-}$ cultad de Ingeniería y los distintos agentes locales, resolviendo problemáticas de interés Público y Privado, generando espacios interdisciplinarios que permitan desarrollar la creatividad y la formulación de ideas, a través del concepto de innovación abierta

\section{Objetivos Específicos:}

- Promover la vinculación de los estudiantes secundarios con la Facultad de Ingeniería.

- Estimular el espíritu emprendedor.

- Generar espacios de investigación creativos e innovadores.

- Estimular el trabajo en equipo, compañerismo y comunicación.

- Formular ideas creativas para problemáticas reales de los sectores socioproductivos locales.

- Generar redes de contacto y oportunidad de desarrollo personal.

- Incentivar el respeto por el medio ambiente, la diversidad cultural y la responsabilidad social empresarial.

En este evento las problemáticas que se presentan pueden ser de varios sectores de actividad o temas diversos: productivos, tecnológicos, sociales, ambientales, organizacionales. Donde las empresas y organizaciones locales deben diseñar estos desafíos centrándose en la búsqueda de Innovación.

Los estudiantes asistentes forman equipos de entre 5 y 10 personas y eligen un desafío que les interese para resolverlo y luego de las 24 hs presentar la solución en un video de 3 minutos. Este video es evaluado por un jurado designado por la organización del evento y son premiados los primeros tres puestos y la institución a la que representan.

Una vez que los estudiantes llegan al evento, se presentan los desafíos y ellos son los encargados de formar los equipos, de la mejor manera que les parezca. Luego eligen la problemática que les interese y con los equipos que formaron comienzan a trabajar 
para poder presentar la solución al finalizar el evento.

Durante toda la jornada, además del trabajo en la solución, a los asistentes se les presentan distintas actividades de integración y capacitación en temáticas que creemos necesarias para su motivación y aprendizaje. Durante la actividad recreativa a los estudiantes se los obliga a salir del lugar de trabajo por media hora, para relacionarse con los demás asistentes mediante actividades propuestas por la organización: Ping Pong, Tejo, Metegol, Básquet, Fútbol tenis, juegos de cartas o simplemente tomar mate entre ellos.

A la noche se realiza el ChoriRock, que es una cena cultural donde se hace una choripaneada para todos los participantes del evento y se invitan bandas de la ciudad para que puedan presentarse y tocar para los asistentes, esta actividad es abierta a la comunidad. Las bandas que se presentaron están integradas por estudiantes de la $\mathrm{Fa}^{-}$ cultad de Ingeniería y por adolescentes de la ciudad, que en algunos casos concurren a las escuelas participantes del evento.

Para las instancias de capacitación se realiza el taller de Creatividad, en el que se presentan conceptos y actividades con la idea de fomentar la creatividad en los estudiantes y aportar a la búsqueda de ideas innovadoras para el desafío elegido. Mientras que en el taller de Modelo de Negocios y Pitching, se presentan los conceptos del modelo de negocios Canvas, enfocándose en la segmentación de los clientes y la propuesta de valor, sumándose el concepto de Pitching, que consiste en cómo poder presentar de forma correcta y completa una idea en poco tiempo.

En adhesión a estas actividades, estu- diantes voluntarios de la Facultad de Ingeniería y miembros de la organización son denominados Tutores de los distintos equipos y se encargan de visitarlos continuamente para evacuar dudas que puedan surgir y brindar asistencia en caso de necesitarla.

\section{METODOLOGÍA}

Las metodologías de actividades de innovación abierta se corresponden con la filosofía de buscar maneras de resolver invitando a pensar soluciones en equipo. La inteligencia colectiva es una realidad y comienzan a aparecer instancias de entrenamiento para explotarla. Para la aplicación de esta metodología se utiliza el modelo de Charrete de trabajo. La traducción de Charrete, del francés, quiere decir "pequeño carro". Cuenta la leyenda que allá por el Siglo XIX en la École des Beaus Arts en Paris, cada fin de ciclo lectivo los profesores de Arquitectura circulaban con esos pequeños carros por los pasillos de las aulas recolectando los trabajos de fin de año de los alumnos, quienes saltaban sobre el carro nuevamente para hacerle modificaciones finales mientras el mismo terminaba su recorrido. La inflexibilidad de la hora de entrega generaba una atmósfera de adrenalina y excitación y se pensaban mejoras hasta último momento para esos dibujos de fin de año.

En este sentido, la novedad que plantea el trabajo es la idea de popularizarlos en el sistema educativo como herramientas para el entrenamiento de competencias emprendedoras.

La aplicación del charrete de trabajo son los rally de innovación, que representan la externalidad del marco conceptual de la innovación abierta. En síntesis, la velocidad del cambio del entorno obliga a las 
instituciones a correr la intensa carrera de la innovación, pero no de manera solitaria, ya que van a necesitar incrementar su capacidad de encontrar, procesar y desarrollar nuevas ideas. Saltaran las fronteras de la organización abriendo paso a buscar las ideas más exitosas allá donde estas se encuentren, no importa si fueron generadas por un grupo de jóvenes en una universidad pública, un equipo de investigadores en un centro del CONICET, una comunidad on-line de usuarios o el grupo de expertos de la organización.

Estos rally de innovación sirven como instancia de entrenamiento de competencias bien puntuales: no se considera un ejercicio de identificación de oportunidades, teniendo en cuenta que el proceso de validación de una idea tiene que ver con el contexto, con el análisis de mercado, acción que no ocurre en veinticuatro horas corridas. Se lo toma como una excelente instancia de entrenamiento para trabajar en equipo en situaciones bajo presión, en un tiempo acotado que marca el ritmo de la entrega y la actividad. Alienta el enfoque multidisciplinario y el entrenamiento en comunicación, teniendo en cuenta que el prototipo a presentar, de poco tiempo de duración tiene que tener tanto respuestas técnicas como de diseño. Es un excelente ejercicio de creatividad, buscando respuestas innovadoras a retos reales planteados por empresas y organizaciones, aplicando competencias emprendedoras como el trabajo en equipo, la búsqueda de información, desarrollo de redes de apoyo y el saber comunicar.

Para la ejecución de este evento y en relación a lo anteriormente mencionado se decidió aplicar esta metodología de Charrete de trabajo, llevado a los estudiantes de escuelas secundarias, apoyado de igual manera en la fomentación y desarrollo de competencias emprendedoras.

Para esto, se contó con la colaboración de la inspectora distrital de escuelas secundarias de gestión pública y la inspectora de escuelas secundarias de gestión privada. En primera instancia se realizaron reuniones con cada Inspectora para presentarles el proyecto, sus alcances y objetivos, luego se compartió esta información con los directores de las distintas instituciones de educación secundaria de Olavarría y la zona.

Se designa al estudiante Joaquín Domato como coordinador y promotor del evento, que se encargó del contacto con las escuelas y la difusión y promoción de éste en las instituciones interesadas.

La difusión se hizo personalmente en las instituciones, con materiales gráficos, describiéndoles a los alumnos la experiencia que se vivirá participando del evento con las diferentes actividades que se realizan durante la jornada.

Durante esta jornada, se realizan actividades de integración para fomentar la relación entre los estudiantes participantes como una actividad recreativa en horas de la tarde y una choripaneada con la presencia de bandas que denominamos ChoriRock. También se realizan instancias de capacitación a cargo de docentes y estudiantes voluntarios, como son el Taller de Creatividad y el Taller de Modelo de Negocios y Pitching. Estos dos talleres son de utilidad para los estudiantes participantes a la hora de realizar el video final con la solución propuesta para ser evaluado.

La evaluación de los videos la hace un jurado compuesto por representantes de las organizaciones que auspician, colaboran y son parte de la organización del evento y 
estudiantes de la Facultad de ingeniería de Olavarría. Este jurado toma la decisión final sobre los ganadores del evento basando en cuatro pilares de evaluación que son:

- Innovación y creatividad

- Análisis de información científica y técnica

- Calidad de la presentación

- Eco - responsabilidad.

Finalmente, los evaluadores asignan puntaje a cada uno de estos pilares y por suma de puntos se definen los ganadores del evento, entregando premios a los ganadores.

\section{RESULTADOS}

En el mes de junio de 2015 se realizó la primera edición del evento, y en octubre se llevó a cabo la segunda edición. En total participaron 147 estudiantes de 14 escuelas de Olavarría y la zona, tanto de gestión pública como privada, presentando 22 soluciones creativas e innovadoras a los 28 desafíos planteadas por empresas y organizaciones locales.

En estos eventos hubo un total de 12 empresas e instituciones públicas que acompañaron económicamente y presentaron los 28 desafíos para que los jóvenes puedan generar propuestas de solución, acompañan el proyecto, Fundación Loma Negra, Losa Olavarría, Inzama Potes S.A., Molino Olavarría, ADELO, Ferrosur Roca, Facultad de Ingeniería Olavarría, Crecer Juntos ( $\mathrm{Ta}$ ller protegido), Tancredi Vigas y Bloques S.R.L., Finning Argentina S.A. - Sucursal Olavarría, Qutral y Eco Control.

En la primera edición invito a participar a todas las escuelas de gestión estatal de Olavarría y la zona, contando finalmente con 11 escuelas inscriptas y 153 estudiantes del último año del nivel secundario. Una vez llegada la fecha asistieron al evento 93 jóvenes, formándose 11 equipos, a decisión de los estudiantes. Algunos de estos equipos estaban conformados por estudiantes de distintas instituciones, logrando la interdisciplinaridad que se buscaba.

\begin{tabular}{|l|l|l|l|}
\hline \multicolumn{2}{|l|}{24} & Hs de innovación FIO 2015 & primera edición \\
\hline Escuelas participantes & No de Inscriptos por escuela & Alumnos que asistieron \\
\hline 1 & ENAPE & 27 & 13 \\
\hline 2 & Recalde (EES NN19) & 2 & 0 \\
\hline 3 & Fonavi (EES No12) & 2 & 2 \\
\hline 4 & Nacional (EES NN66) & 25 & 17 \\
\hline 5 & Sierra Chica (EES NN17) & 8 & 4 \\
\hline 6 & Escuela de Educación Agropecuaria & 11 & 11 \\
\hline 7 & EES No20 (Fray Romeo Musaragno) & 14 & 3 \\
\hline 8 & EES No16 (Barrio Jardín) & 13 & 8 \\
\hline 9 & Loma Negra (EES No3) & 14 & 13 \\
\hline 10 & EEST No2 (Ex Industrial) & 24 & 15 \\
\hline 11 & EES No1 (Bancario) & 13 & 7 \\
\hline & Total & 153 & 93 \\
\hline
\end{tabular}


En esta Jornada los equipos eligieron los desafíos que les interesaban y presentaron su solución propuesta en un video, creándose 15 videos, ya que un equipo decidió elegir cuatro desafíos a resolver.

Para la segunda edición, se optó por convocar a las escuelas secundarias de gestión privadas, para así lograr la participación de todas las escuelas. En esta ocasión 6 escuelas se mostraron interesadas a participar. $\mathrm{Al}$ cerrarse la inscripción al evento se contó con 99 estudiantes inscriptos en total, asistiendo finalmente 59 jóvenes.
Además participaron estudiantes de séptimo año de la EEST No2 (Ex Industrial) que se acercaron a nosotros en reiteradas veces con la intención de participar en este evento.

En esta ocasión se formaron siete equipos logrando nuevamente que muchos de estos estén conformados por estudiantes de distintas escuelas. Como resultado se presentaron siete videos como propuesta de solución a los desafíos elegidos.

\begin{tabular}{|l|l|l|l|}
\hline \multicolumn{4}{|l|}{24 Hs de innovación FIO 2015 Segunda edición } \\
\hline Escuelas participantes & No de Inscriptos por escuela & Alumnos que asistieron \\
\hline 1 & Colegio Nuestra Señora de Fátima & 27 & 0 \\
\hline 2 & Colegio Privado Nuevas Lenguas & 14 & 2 \\
\hline 3 & Instituto San Antonio de Padua & 31 & 31 \\
\hline 4 & Mariano Moreno & 10 & 9 \\
\hline 5 & EEST No2 (Ex industrial) & 5 & 5 \\
\hline 6 & Instituto Privado Sierras Bayas & 12 & 12 \\
\hline & Total & 99 & 59 \\
\hline
\end{tabular}

Los resultados cuantitativos fueron expuestos más arriba, pero hay mucho logro cualitativo de esta iniciativa difícil de mostrar en palabras. Además de contar con el compromiso de todas las instituciones $y$ empresas participantes, se puede destacar la actuación de los jóvenes es lo más importante.

Durante estas 24 hs que los estudiantes pasan en complejo de la Facultad de Ingeniería de Olavarría, se observa, además de conductas intachables y diversión, aprendizaje colaborativo y continuo, principalmente de la interrelación de estos con sus compañeros y miembros de la FIO, como también de la experiencia adquirida en el desarrollo de la solución y búsqueda de in- formación para resolver la problemática de la mejor manera que consideran.

Se puede destacar además el trabajo en equipo, compromiso con la tarea, sana competencia, compañerismo, creatividad, responsabilidad, dedicación e intercambio, como algunas de las competencias emprendedoras que pudieron ponerse de manifiesto, mostrando un aprendizaje de los jóvenes en la jornada.

A continuación se empieza a trabajar con las ideas participantes para acompañarlas en su desarrollo a proyectos y su evaluación a través del Centro de Desarrollo Emprendedor e Innovación, recientemente creado por la Facultad de Ingeniería de Olavarría. 
En este sentido, de la primera edición se dos equipos se presentaron para continuar con el proyecto, a los que se los acompañó durante dos meses en el desarrollo. Hoy en día estos proyectos se encuentran en receso, con la promesa de ambas partes de continuarlo ni bien sea posible.

De los proyectos logrados en la segunda edición, se están realizando reuniones con uno de los equipos participantes para poder continuar con el desarrollo y estudio de la idea.

\section{Evidencias, mails recibidos desde las es- cuelas:}

$\varnothing$ No los pude acompañar personalmente porque estuve bastante complicado en esos dos días. Pero se que los chicos la pasaron muy bien y me gustarías que sepas que se la jugaron ustedes por lo nuevo $y$ distinto y yo también, porque era mucha responsabilidad y nos pidieron lección paseo y yo me puse como responsable. Si pasaba algo con mis alumnos, yo era el responsable directo. Pero a pesar de ello, confiaba en la propuesta porque se que a los chicos de Sierra Chica les hace bien estar con otros aprendiendo.

Abrazo.

José Mogávero

Director $\mathrm{EESN}^{\circ} 17$

-

$\varnothing$ Gracias, los chicos volvieron muy felices por la experiencia. Alicia Rota EES No 16.

.

$\varnothing \quad$ Muchas Gracias a Uds por la invitación, la encuesta y las opiniones de nuestros alumnos reflejan lo importante que fue para ellos y para la escuela, la experiencia vivida.

También es un estímulo para mejorar nuestras propuestas de enseñanza.

El próximo año estaremos nuevamente. Gracias! Saludos!

Equipo Directivo de E.E.S.N ${ }^{\circ} 6$

$\varnothing$ Marcos, te felicito!!

Realmente la alegría y entusiasmo de los chicos pocas veces lo ví. Eugenia Berrueta, Escuela Agropecuaria

$\varnothing \quad$ Gracias Marcos: fue una gran experiencia para ellos y muy enriquecedora. Estaremos acompañando el próximo año.

Saludos

Karina Elbey.

Inspectora de Nivel Secundario de Gestión Privada

$\varnothing$ Gracias! Muy linda experiencia, la pasamos muy bien.

Atte.

Liliana Rollheiser.

Directora. Esc Sec M. Moreno.

$\varnothing \quad$ Estimados/as :

Muchas gracias por la invitación a participar de la propuesta y porenviarlos resultados de la encuesta.

Esperamos poder participar también el año que viene. 
La experiencia fue muy buena.

Saludos cordiales

Lic. Gloria Elida Scrimizzi

Vicedirectora

Instituto Privado "San Antonio de Padua" tes:

Opiniones de Estudiantes participan-

Agostina Arata - Alumna del Instituto San Antonio de Padua, Orientación Arte Artes visuales:

"Me encanto poder ir a la facultad, me gusta el clima que hay entre ustedes. En el secundario la vemos siempre con miedo a la facultad, como que va a ser todo serio y aburrido y al final me di cuenta que no es asi"

Nahuel Mondotegui - Alumno de EEST No2 (Ex Industrial), Orientación Técnico Electromecánico:

"La experiencia estuvo muy buena, fue interesante, fue bastante agradable porque bubo bastantes chicos que participaron. Mucha variedad de problemáticas y lo bueno es que eran de temas regionales. Podias innovar y expresarte a tu manera e intercambiar opiniones con otros chicos y ayudarse entre si"

Facundo Benítez - Alumno del Instituto San Antonio de Padua, Orientación Arte - Artes visuales:

"Me pareció una experiencia fantástica, fue muy divertida. Aprendimos un montón y pudimos compartir con nuestros compañeros y con gente que no conocíamos. Aprendimos a sociabilizar con los demás, a trabajar en equipo, a dividirnos el trabajo y en vez de echarnos la culpa, seguir trabajando sobre eso y encontrar la solución. Muy recomendable."
Carla Almada - Alumna del Colegio Privado Mariano Moreno - Orientación Ciencias Sociales:

"Me pareció una experiencia única, muy buena. Nunca habiamos asistido a un evento de tantas horas seguidos y eso bizo que sea diferente. Trabajamos mucho durante toda la noche pero a pesar del cansancio salieron ideas y cosas muy productivas. La atención de la organización fue muy buena y siempre bubo buena predisposición de ellos."

\section{Registro en imágenes de los eventos:}
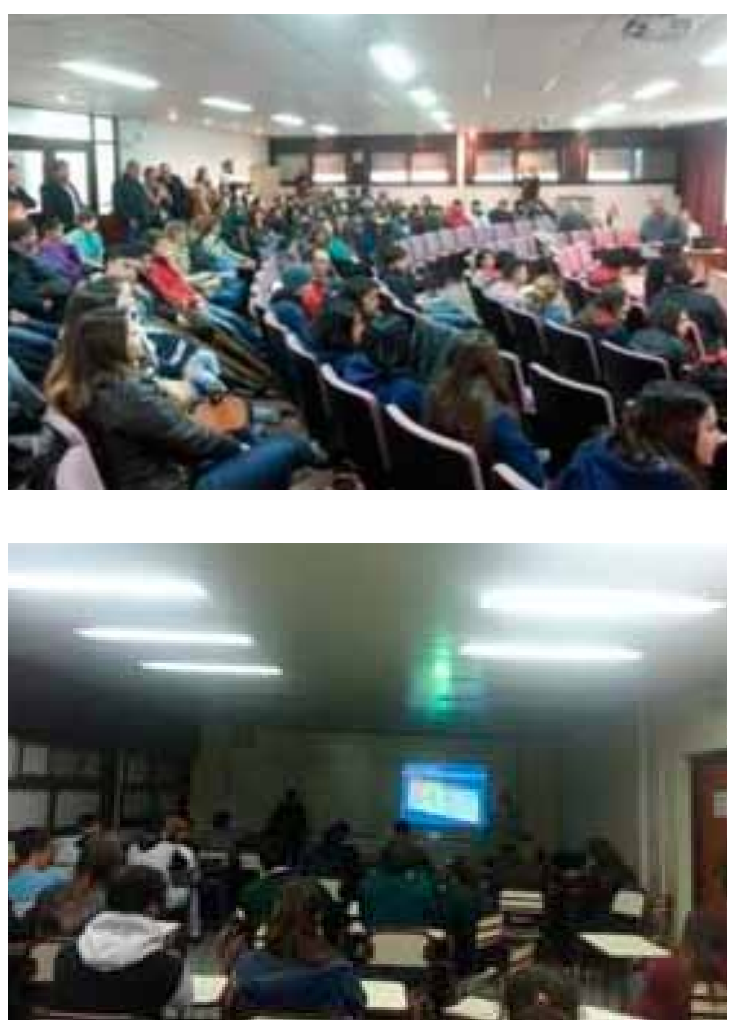

\section{CONCLUSIÓN}

En el sentido de este trabajo se concluye que la metodología de Innovación Abierta, puesta en práctica con estudiantes de la Facultad de Ingeniería de Olavarría, y luego llevada a los estudiantes de la escuela secundaria, es una herramienta de aprendiza- 


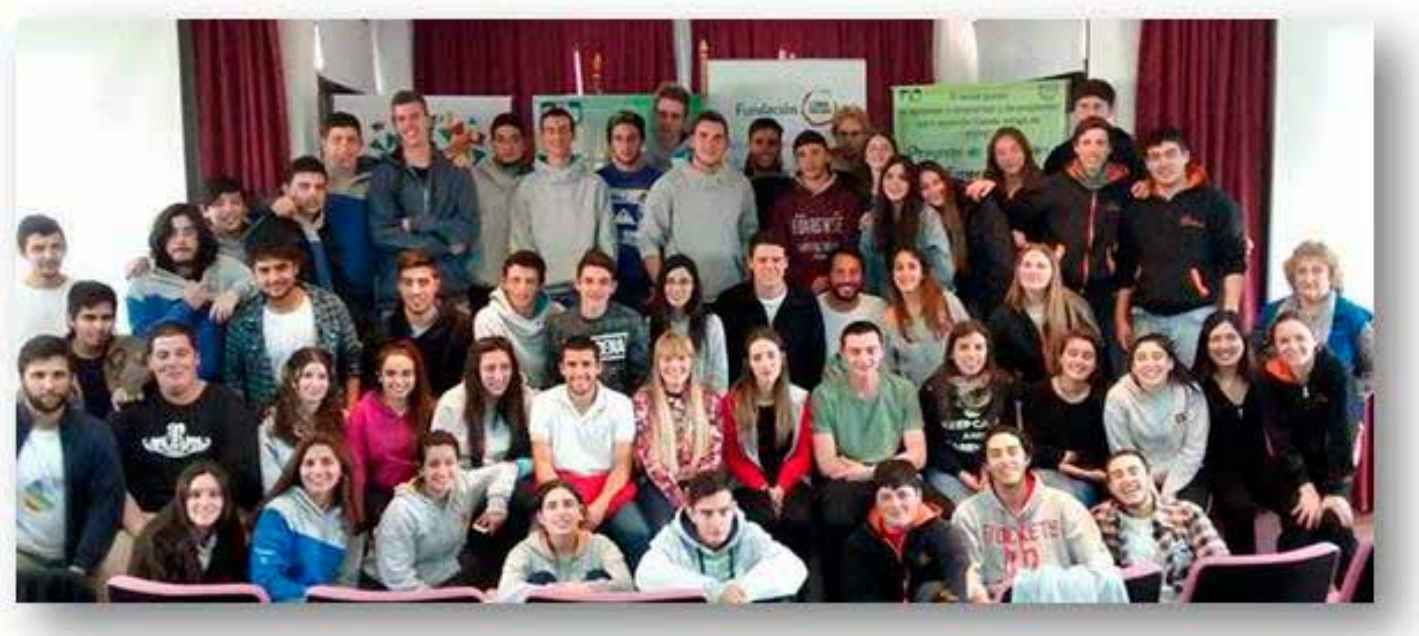

je vivencial que logra motivar y con la que se obtienen los resultados que esperamos en los jóvenes.

Esta metodología de Innovación, dentro y fuera del aula, contribuye en parte, a lograr el estado de flujo al que hace referencia Csikszentmihalyi, motivando al joven con problemáticas reales de su entorno, y desafiantes, tratando de eliminar el desgano y ansiedad.

Además hemos contemplado que en este sentido, no es necesario que a los estudiantes se les enseñe dentro de un aula con "problemas juguete", sino que al desafiarlos con problemáticas reales donde la búsqueda de la solución dependa enteramente de ellos, se logran resultados superiores. Esto se justifica en el ciclo de Kolb, donde la experiencia vivida genera aprendizaje, no solo sobre el problema que se resuelve, sino también, los jóvenes adquieren competencias para su desarrollo tanto en la formación académica como en su rol profesional. Cumpliendo así, con la política previamente mencionada, del fomento de la cultura emprendedora y desarrollo de competencias en este sentido.

Además entendemos que el fortalecimiento de los vínculos interinstitucionales, entre las organizaciones partícipes de la metodología, y con los distintos sectores de la comunidad contribuye al desarrollo local y con él a la asimilación de las competencias emprendedoras que esperamos que adquiera nuestra población objetivo, los jóvenes.

Esta metodología de eventos en el ámbito de la enseñanza secundaria, debe ser sistematizado e instalada, transformándose así en una innovación en el ámbito del nivel secundario. Las devoluciones recibidas por los docentes y directivos, nos invita a pensar estrategias para continuar y fortalecer estas actividades. El aprendizaje vivencial, basado en problemas reales y con posibilidad de generar conocimiento compartido es una herramienta de mucho valor académico si los docentes se involucran en todo el proceso en un rol de facilitadores. A partir de los resultados que se obtengan debieran iniciarse instancias de proto-investigación que conduzcan a nuevas acciones en clave de integralidad (docencia - investigación extensión). El impacto de los resultados de estos procesos es inmediato si existe la decisión política y los apoyos institucionales que promuevan distintas formas de incorporar conocimientos y adquirir competencias, transgrediendo lo instituido. 
Esto no se logra en soledad, es imprescindible la construcción de redes interinstitucionales que, junto al sector privado, generen la sinergia necesaria para potenciar las acciones de los actores del ecosistema emprendedor. Las jornadas organizadas por nuestra Facultad han logrado resultados muy positivos en este sentido, desde el apoyo financiero, hasta la declaración de interés municipal del gobierno local.

Entendemos que este es uno de los caminos hacia la innovación empresaria, pública y educativa. 